\title{
Multipath Transport Protocol for Heterogeneous Multi-homing Networks
}

\author{
Younghak Hwang, Brownson O. Obele, and Hyuk Lim* \\ Gwangju Institute of Science and Technology (GIST), Korea \\ \{yhhwang, brownson, hlim\}@gist.ac.kr
}

\begin{abstract}
We present a new multipath transport protocol - Heterogeneous Multipath Transport Protocol (HMTP), powered by fountain codes to improve throughput performance and path utilization of heterogeneous multi-homing networks. HMTP solves the receive buffer blocking problem and eliminates the need for retransmission and in-order packet delivery, especially in a heterogeneous multi-homing network.
\end{abstract}

\section{Keywords}

Multi-homing, receiver blocking, fountain codes

\section{INTRODUCTION}

In recent years, due to advances in hardware design and technology, it is now common to find Internet hosts with multiple network interfaces such as Ethernet, WiFi, and $3 \mathrm{G}$. Nonetheless, most of these hosts are connected to network services through only one interface at a time. If these hosts simultaneously establish network connections using multiple interfaces, their performance would be considerably improved.

In order to gainfully exploit the multipath diversity from multiple network connections, a new transport protocol that can reliably and efficiently distribute data packets through multipath is highly desired. Most existing multipath transport protocols are based on TCP [1] and SCTP [2], which rely on ARQ schemes for reliable data transfer. Under heterogeneous multipath environments, their performance decreases due to the "receive buffer blocking problem" in [2]. When a sender transmits a series of packets across multiple paths, out-oforder packets may arrive at the receiver due to the heterogeneous characteristics of multipaths. In this case,

Permission to make digital or hard copies of all or part of this work for personal or classroom use is granted without fee provided that copies are not made or distributed for prof $\mathrm{t}$ or commercial advantage and that copies bear this notice and the full citation on the f rst page. To copy otherwise, to republish, to post on servers or to redistribute to lists, requires prior specif c permission and/or a fee.

ACM CoNEXT Student Workshop, November 30, Philadelphia, USA.

Copyright 2010 ACM 978-1-4503-0468-9/10/11 ...\$10.00. the delivery of the received packets to the upper layer is delayed until all the packets are received.

To overcome this problem, we propose a new multipath transport protocol - HMTP, which is based on fountain codes. Fountain codes [3] are forward error correction (FEC) codes for erasure channels. The fountain encoder endlessly generates new encoded packets by a modulo-2 addition of randomly selected original packets. Regardless of the order in which encoded packets are received, if a sufficient number of encoded packets are collected, the fountain decoder can recover the original packets [3]. Exploiting this feature of fountain codes, HMTP enables a sender to reliably and efficiently transmit packets through heterogeneous multipaths despite heterogeneous loss characteristics of different paths. It also make it possible for a receiver to correctly recover the original data only if a sufficient number of packets are received regardless of their arrival order.

\section{PROPOSED TRANSPORT PROTOCOL}

The proposed algorithm consists of a fountain layer and a transport layer. In HMTP, the transport layer is wrapped with the fountain layer. A multi-homed sender takes $k$ original data packets from a data stream of application layer and then encodes them into independent fountain-encoded packets. These fountainencoded packets are concurrently transmitted over heterogeneous multipaths. Because the packets are transmitted over different paths, some may arrive at the receiver out-of-order with some missing packets due to heterogeneous characteristics (such as loss rate, end-toend delay etc.) of paths. In existing multipath protocols, these out-of-order packets cannot be delivered to the application layer until the lost packets are requested and retransmitted, resulting in increased transmission delays. In HMTP, the fountain-encoded packets received at the receiver are decoded and delivered immediately to the application layer regardless of their arrival order. When $k(1+\varepsilon)$ fountain-encoded packets have arrived, the original data packets can be successfully recovered. Here, $\varepsilon$ is a small positive real number 


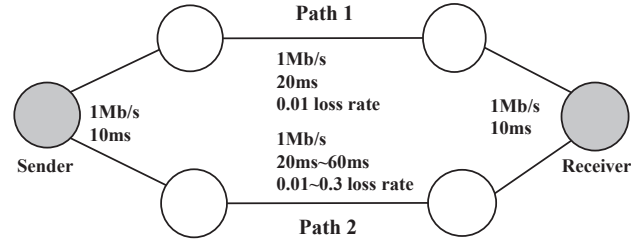

Figure 1: Simulation topology

and is called decoding inefficiency [3]. The detailed operational procedure of the proposed heterogeneous multipath transport protocol is as follows:

(1) At the multi-homed sender, a continuous new-data stream from the application layer is divided into data chunks with $k$ data packets.

(2) The packets in each chunk are encoded into independent fountain-encoded packets.

(3) The header of a fountain-encoded packet includes the chunk ID and the seed for the pseudo-random number generator.

(4) The fountain-encoded packets are delivered to the transport layer and then are continuously transmitted over multiple paths.

(5) At the receiver, the received fountain-encoded packets are delivered to the fountain layer regardless of their arrival order.

(6) The fountain decoder reconstructs the original data chunk and delivers it to the application layer. Then, the receiver sends a stop message to the sender.

The proposed HMTP can be easily implemented by disabling retransmission for in-order delivery of a ARQ scheme of TCP. However, unlike TCP sender, when a packet is lost, it simply generates a new fountainencoded packet and transmits it instead of retransmitting the previously lost packet.

\section{PERFORMANCE EVALUATION}

In order to evaluate the performance of the HMTP, we have conducted simulations using NS-2 simulator [4] and compared its performance against CMT-SCTP with the retransmission policy of RTX-CWND [2]. For HMTP, the parameters of $\delta$ and $k$ for fountain layer are set 0.6 and 1000 , respectively. Once the receiver receives 1095 fountain-encoded packets, it can reconstruct 1000 original packets [3]. Fig. 1 shows the simulation topology. The bandwidth is $1 \mathrm{Mbps}$ on both paths. While the end-to-end delay and loss rate on path 1 are kept constant, for path 2 they are varied from 40 to $80 \mathrm{~ms}$ and from 0.01 to 0.3 , respectively.

We compare the throughput performance of CMTCWND and HMTP when the loss rate of path 1 is

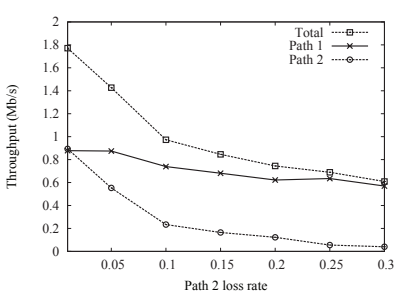

(a) CMT-CWND

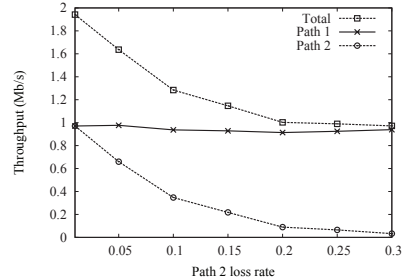

(b) HMTP
Figure 2: Throughput results for receive buffer $=64 \mathrm{~kb}$, path 1 loss rate $=0.01$, and end-to-end delays on both paths $=40 \mathrm{~ms}$

fixed at 0.01 and that for path 2 is varied from 0.01 to 0.3. Fig. 2(a) and (b) show the throughput of CMTCWND and HMTP, respectively. For CMT-CWND, the throughput of path 1 decreases as the loss rate of path 2 increases as shown in Fig. 2(a). When the loss rate of path 2 is high, although the loss rate of path 1 is fixed, the throughput of path 1 decreases due to the receive buffer blocking caused by a lot of lost packets on path 2 . To the contrary, the throughput of path 1 for HMTP is maintained at about $1 \mathrm{Mb} / \mathrm{s}$ in Fig. 2(b). It implies that the throughput of path 1 for HMTP is not affected by the loss rate of path 2 .

\section{CONCLUSIONS}

The main objective of our algorithm is to improve the overall network performance by solving the receive buffer blocking problem. Towards this objective, we have proposed a new multipath transport protocol HMTP, which is based on fountain codes. The proposed algorithm can reliably and efficiently transmit packets through heterogeneous multipath in multi-homing networks. The simulation results show that our proposed transport protocol achieves improved throughput performance and high path utilization.

\section{ACKNOWLEDGMENTS}

This work was supported by the Korea NRF Grant K20901000004-09E0100-00410.

\section{REFERENCES}

[1] C. Huitema. Multi-homed TCP. draft-huitema-multi-homed-01, 1995.

[2] J. Iyengar et al. Performance implications of a bounded receive buffer in concurrent multipath transfer. Computer Communications, 30(4):818-829, 2007.

[3] D. MacKay. Fountain codes. IEE Proceedings Communications, 152(25):1062-1068, 2005.

[4] ns-2 documentation and software, version 2.34. [Online] Available: http://www.isi.edu/nsnam/ns. 